\title{
Once-daily Preservative-free Topical Anti-glaucomatous Monotherapy - A Better Approach?
}

\author{
Toby S Al-Mugheiry ${ }^{1}$ and David C Broadway ${ }^{1,2}$ \\ 1. Norfolk and Norwich University Hospital NHS Foundation Trust, Norwich, UK; 2. School of Pharmacy, University of East Anglia, Norwich, UK
}

DOI: https://doi.org/10.17925/EOR.2020.14.1.21

$\mathrm{F}$ or many years, the mainstay of medical treatment for glaucoma has utilised a variety of topical therapies. Historically, topical medications have been fortified with a variety of agents known as preservatives. Unfortunately, although preservatives are potentially useful, they can also cause an array of adverse effects, impacting on the safety and tolerability of these drugs. The unwanted effects of topical medication preservatives have been identified in both human and animal studies. Here we review the potential advantages of utilising preservative-free (PF) prostaglandin monotherapy as a treatment option for patients with primary open-angle glaucoma. We propose that the use of PF topical anti-glaucomatous monotherapy could improve the management of glaucoma, which is a global cause of visual loss and has a significant impact on healthcare resources.

\section{Keywords}

Glaucoma, preservatives, preservative-free treatment, prostaglandin antagonists, adverse effects

Disclosures: Toby S Al-Mugheiry and David C Broadway have no financial or non-financial relationships or activities to declare in relation to this article.

Review Process: Double-blind peer review.

Compliance with Ethics: This study involves a review of the literature and did not involve any studies with human or animal subjects performed by either of the authors.

Authorship: The named authors meet the International Committee of Medical Journal Editors (ICMJE) criteria for authorship of this manuscript, take responsibility for the integrity of the work as a whole, and have given final approval for the version to be published.

Access: This article is freely accessible

at touchOPHTHALMOLOGY.com

(c) Touch Medical Media 2020

Received: 29 October 2019

Accepted: 9 March 2020

Published Online: 22 July 2020

Citation: European Ophthalmic Review. 2020;14(1):21-5

Corresponding Author: David C Broadway, Department of Ophthalmology, Norfolk and Norwich University Hospital NHS Foundation Trust, Colney Lane, Norwich, Norfolk, NR4 7UY, UK.

E: david.broadway@nnuh.nhs.uk

Support: No funding was received in the publication of this article.
Glaucoma causes an optic neuropathy and is one of the leading causes of blindness globally. Pathologically, patients with glaucoma typically develop irreversible characteristic field defects due to loss of retinal ganglion cells and subsequent characteristic optic nerve-head damage. The main risk factor for development of glaucoma is intraocular pressure (IOP), reduction of which is targeted by mainstay therapies.

Treatment of manifest glaucoma, or significant ocular hypertension, can involve medical, laser or surgical interventions, all of which aim to reduce IOP. Currently, for the majority of patients, medical therapy is utilised for controlling IOP and reducing the risk of progressive visual field deterioration. A variety of oculo-hypotensive agents are available and these can be used alone or in combination. Prostaglandin analogues have been shown to be superior to $\beta$-adrenergic receptor blockers, $\alpha$-adrenergic agonists or topical carbonic anhydrase inhibitors, in lowering IOP. ${ }^{2,3}$ Prostaglandin analogues, require only once-daily dosing, making them a popular choice to reduce IOP, with a good adherence record. Prostaglandin analogues have a good safety profile and are recommended as a first-line treatment for glaucoma by the European Glaucoma Society Guidelines. ${ }^{4,5}$

Unfortunately, all topical anti-glaucomatous agents, including prostaglandin analogues, can induce adverse effects leading to poor adherence. ${ }^{6}$ Topical therapy-induced ocular toxicity, ocular/periocular allergy or systemic side effects, may result in such a therapy being inappropriate and/or ineffective. Minimising adverse effects and in particular ocular/periocular toxicity/allergy can be achieved through the use of preservative-free (PF) agents, since it is frequently the preservative, rather than the drug itself, that induces such adverse effects.

\section{Use of topical therapy preservatives and ocular toxicity}

Topical anti-glaucomatous agents contain a variety of compounds besides the active drug; these include preservatives, stabilising components and buffers. Preservatives are potentially useful in that they prevent the growth of microbes, prolong shelf-life and degradation of the active drug. ${ }^{\text {A }}$ variety of preservatives may be used in bottles of topical therapeutic agents and these include benzalkonium chloride (BAC), benzododecinium bromide, purite, polyquaternium-1 and cetrimide. The most commonly used preservative in ophthalmic products is BAC, which is used in concentrations ranging from $0.004-0.02 \%$. BAC is a quaternary ammonium surfactant that acts as a cationic detergent binding to cell membranes and causes cell lysis, the latter being the mechanism by which it kills microbes. Unfortunately, the toxic effects that BAC have on microbes, may also affect human cells, resulting in increased oxidative stress and apoptosis. ${ }^{8}$ It has been known for many years that BAC can cause ocular toxicity in both humans and in animal models. ${ }^{9}$

Compared with other monotherapeutic options, including $\beta$-blockers, $\alpha_{2}$-adrenergic agonists and carbonic anhydrase inhibitors, prostaglandin monotherapy seems to be the most efficacious at 


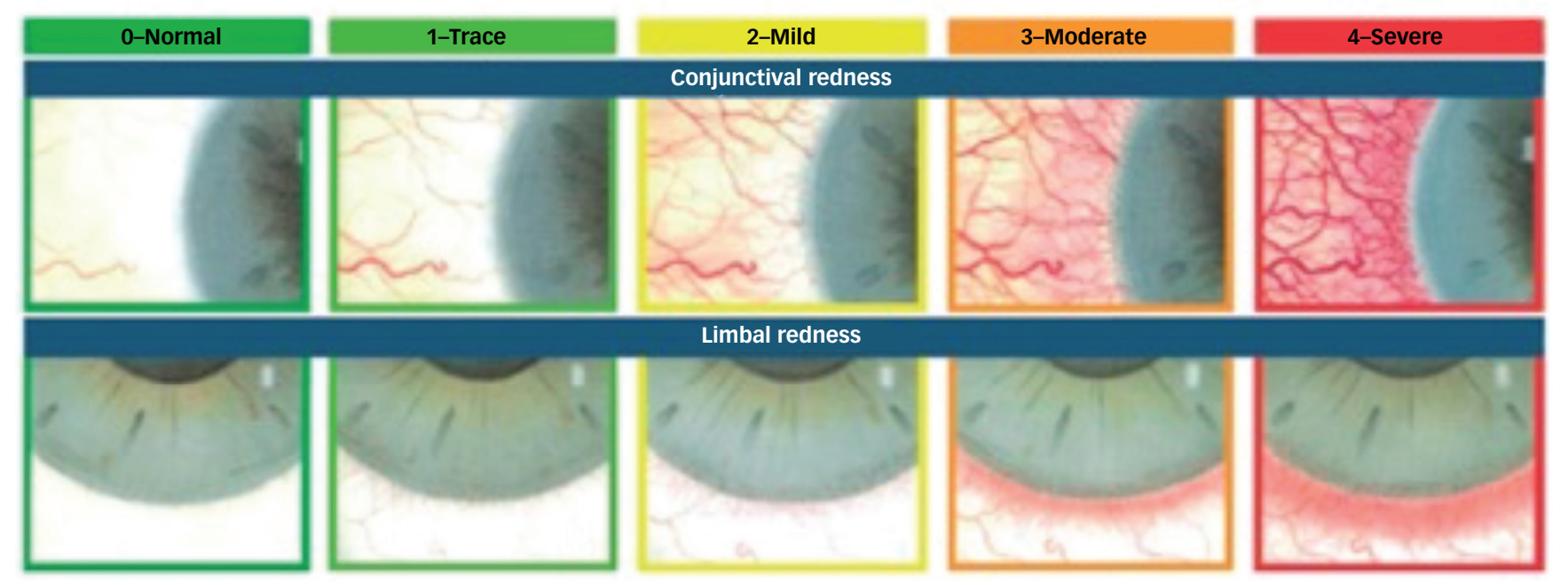

Reproduced with permission from Prof. Nathan Efron. ${ }^{26}$

lowering IOP.10 Moreover, prostaglandin monotherapy is more likely to achieve a target IOP (e.g., <18 mmHg) than any other monotherapy, and is comparable to some dual therapies. ${ }^{10}$ The prostaglandin analogues that have been used to lower IOP in clinical practice include latanoprost, travoprost, bimatoprost, tafluprost and unoprostone. A systematic review and meta-analysis of 114 randomised controlled trials showed all of these agents to be effective at lowering IOP at 3 months compared with placebo, with bimatoprost, travoprost and latanoprost being the most effective." Latanoprost was the first prostaglandin analogue introduced for the treatment of glaucoma and ocular hypertension and is frequently used as a first-line therapy. ${ }^{12}$ Latanoprost binds to its prostaglandin receptor, $\mathrm{PGF}_{2 \alpha^{\prime}}$ causing an increase in uveoscleral aqueous humour outflow and a lesser effect on trabecular outflow, leading to a reduction in IOP. Latanoprost is comparable to other prostaglandin analogues in efficacy and indeed, a multicentre randomised interventional study of 411 patients showed latanoprost had a similar reduction in IOP compared with bimatoprost or travoprost. ${ }^{13}$ In addition, latanoprost causes a greater reduction in IOP compared with unoprostone ${ }^{14,15}$ or tafluprost. ${ }^{16}$ Although a study has shown bimatoprost to be slightly more efficacious in lowering IOP, the same study also reported that latanoprost caused less conjunctival hyperaemia compared with bimatoprost.17

One of the main drawbacks of preserved anti-glaucomatous therapy, however, is the ocular toxicity that they produce. Both the active ingredient and preservatives may cause this. One preservative, BAC, can cause symptomatic clinical or sub-clinical changes to the ocular surface despite providing useful antimicrobial and stabilising properties. At a cellular level, BAC increases oxidative stress through the production of inflammatory mediators and this has been shown to lead to apoptosis of conjunctival cells. ${ }^{18,19}$ Sub-clinical sub-conjunctival inflammation and eventual fibrosis can develop following several years of chronic administration of preserved drops, due to an increase in inflammatory cell and fibroblast numbers within the conjunctiva. These changes can lead to failure of subsequent trabeculectomy, ${ }^{20}$ reduced inferior conjunctival fornix depth (indicative of a fibrotic reaction) ${ }^{21}$ and even symblepharon formation.22 Even at low concentrations, BAC can cause trabecular meshwork toxicity and apoptosis. ${ }^{23}$ The adverse effect that BAC can have on the trabecular meshwork may well accelerate the glaucoma by impairing its function, potentially making its administration counterproductive, at least in part.
It should be noted that the prostaglandins have similar concentrations of BAC, yet some cause more side effects than others, for example, in one study, travoprost, caused more redness; this could be interpreted to be a result of the active ingredient. ${ }^{24}$ Another study, however, has reported that bimatoprost caused more hyperaemia than travoprost. ${ }^{25}$ When prescribing a prostaglandin, a clinician should always be aware of the side effects of prostaglandin analogues in general, which includes, but is not limited to, the development of conjunctival hyperaemia (Figure 1), ${ }^{26}$ hypertrichosis, iris dyschromia, peri-ocular skin pigmentation and cystoid macula oedema. It should be noted that all topical medication side effects may result in poor adherence, and therapy that is not used appropriately is destined to fail with respect to efficacy (Figure 2). Early hyperaemia due to the active agent in prostaglandin analogue therapy is particularly marked in the first few days of administration but tends to reduce thereafter. ${ }^{27}$

\section{Effect of drop preservatives on the conjunctiva, cornea and tear film}

The use of preservatives is well documented to have adverse effects on the ocular surface including inducing hyperaemia, tearing, burning, irritation and pain in a proportion of patients. ${ }^{28,29}$ The adverse effects on the ocular surface have been shown to be worse with preserved drops in comparison with PF drops. In one study, drop preservative was implicated as the direct culprit for the side effects, since switching from preserved to PF treatment, there were reduced reports of adverse effects and improved tolerance..$^{29}$ The majority of patients with glaucoma are elderly with a higher incidence of meibomian gland dysfunction (blepharitis), dry eye and a high tear evaporation rate, making them more susceptible to the side effects of preserved treatment. In addition, it has been shown that use of preserved anti-glaucomatous medication may lead to an alteration in corneal sensitivity and a loss of superficial epithelial cells..$^{30}$

BAC also has a toxic effect on conjunctival goblet cells, decreasing mucin production, which severely disrupts the structure of the pre-corneal tear film. Furthermore, toxic follicular conjunctivitis can cause loss of microvilli on surface epithelial cells with loss of contact between cells, resulting in desquamation and ulceration. ${ }^{31}$ Insertion of BAC into cell membranes allows gap formation and ionic imbalances, leading to osmotic changes, oedema and cellular damage. ${ }^{32}$ BAC also leads to the generation of superoxide anions, leading to apoptosis of cells. ${ }^{8}$ 
Figure 2: Ocular toxicity and intolerance leads to poor adherence and poorly managed glaucoma

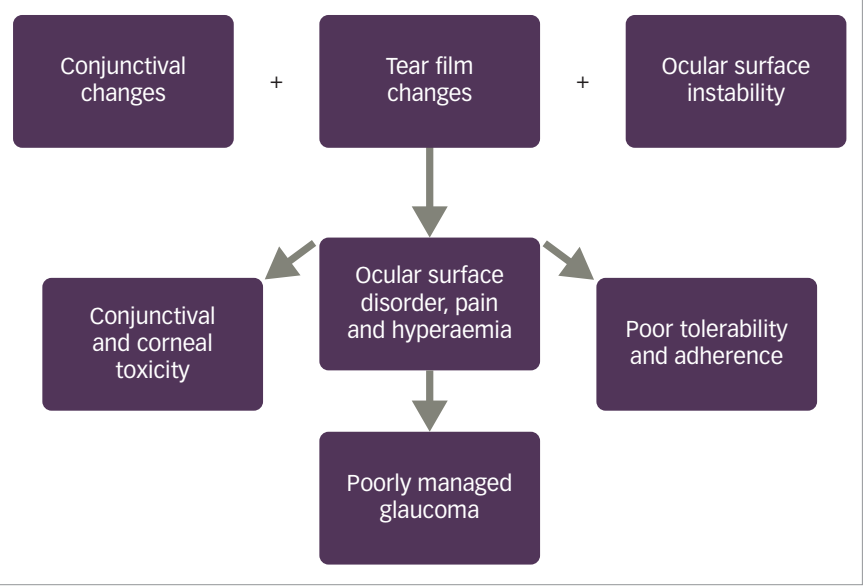

Moreover, a small concentration of BAC $(0.01 \%)$ can cause rupture of the corneal epithelial cells, risking the development of epithelial erosions and an infiltration of inflammatory cells into the conjunctiva. ${ }^{33}$ The effects of glaucoma medications on meibomian gland function is also important. Indeed, a recent study showed preservative-containing prostaglandin analogues caused severe meibomian gland loss, as well as more ocular discomfort and a poorer ocular surface compared with PF formulations. ${ }^{34}$

The use of BAC can impair tear film function, reducing tear film break-up time and inducing changes in both the mucus-layer and the lipid-layer. ${ }^{35}$ The dispersing detergent effect of BAC results in loss of the lipid-layer of the pre-corneal tear film, undue evaporation of the aqueous-layer, subsequent instability of the overall tear film and sometimes the precipitation of the mucus-layer. In predisposed patients, persistent administration of preserved drops can eventually result in the development of a symptomatic ocular surface disorder, which may, in severe cases, lead to corneal erosions and ulceration. In animal studies, it has been shown in rabbits that an increasing concentration of BAC causes a greater number of corneal micro-lesions and increases the production of markers of corneal damage (release of lactate dehydrogenase and albumin). ${ }^{36}$ Moreover, a separate study showed a $0.01 \%$ concentration of BAC delayed corneal healing whilst $0.02 \%$ completely inhibited the healing process post-keratectomy in rabbit eyes. ${ }^{37}$

Of particular importance with respect to the management of the glaucoma itself, long-term administration of combination therapy has been shown to have a detrimental effect on the outcome of glaucoma filtration surgery (trabeculectomy); this may be due to the effects of preservatives on the conjunctiva and the ocular surface through promoting a chronic inflammatory response and enhancing fibroblast activity during the post-operative period of wound healing. ${ }^{38}$

\section{The advent of preservative-free monotherapy}

Adverse effects of preservatives can be detrimental to the tolerability of glaucoma medication, which can lead to poor adherence. Indeed, a large systematic review conducted over a 40-year period showed that poor compliance and persistence was a common occurrence in patients on glaucoma therapy and this, at least in part, may well have been due to the administration of preservatives within anti-glaucomatous therapy. ${ }^{39}$ Adverse effects relating to the administration of preserved topical therapy have been identified as a primary reason for lack of adherence with anti-glaucomatous therapy. ${ }^{40}$ Poor adherence with therapy can, of course, lead to sub-optimal treatment of glaucoma with uncontrolled IOP, poor visual outcomes and/or reduced quality of life.

It has been shown, in a large European multicentre study involving 9,658 patients, that switching from preserved to PF drops leads to a reduction in signs and symptoms associated with ocular surface disorder such as pain or discomfort during instillation (48\% versus 19\%), foreign body sensation (42\% versus 15\%), stinging or burning (48\% versus $20 \%$ ) and dry eye sensation (35\% versus $16 \%$ ). ${ }^{41}$ Furthermore, patients on the PF treatment were $50 \%$ less likely to report ocular surface side-effects.

\section{Efficacy and tolerability of preservative-free monotherapy}

The first PF prostaglandin analogue to become available was tafluprost (SaflutanTM, Santen, Osaka, Japan) in 2008. A large study of over 500 patients showed that using PF tafluprost either as a substitution for the fixed or non-fixed combination, as an add-on to the existing combination therapy or as one agent in a newly initiated treatment regimen, reduced IOP effectively whilst being better tolerated with fewer ocular signs or symptoms. ${ }^{42}$ Indeed, PF tafluprost has been shown to lower IOP effectively in several different populations in patients with primary open-angle glaucoma (POAG) or normal tension glaucoma. ${ }^{43-45}$ In a meta-analysis of two phase IIIb clinical trials involving 339 patients, switching from BAC-preserved latanoprost to PF tafluprost kept IOP at the same level whilst reducing ocular signs and symptoms. ${ }^{46}$ Several studies have emphasised that as well as decreasing IOP significantly, PF tafluprost is also very well tolerated in patients with POAG, normal tension glaucoma, ocular hypertension and pseudo-exfoliation glaucoma. .7.48 $^{\circ}$ Other agents to consider include PF bimatoprost (Lumigan ${ }^{\oplus}$, Allergan, Dublin, Ireland; or Eyreida ${ }^{\circledR}$, Aspire Pharma, Hampshire, UK), which has also been shown to be effective as demonstrated in a large study of 1,830 patients in which mean IOP was reduced by $23 \% .49$

In addition, a large multicentre prospective historical control study of 691 patients showed that patients switched from BAC-preserved bimatoprost or BAC-preserved latanoprost to BAC-free travoprost (preserved with polyquaternium-1), demonstrated a significant reduction in symptoms secondary to ocular surface disease, improved IOP control and reduced conjunctival hyperaemia. Of note, $72.4 \%$ of patients preferred BAC-free travoprost compared with prior therapy, indicating that BAC is a cause of adverse symptoms, at least in comparison with polyquaternium-1.50 Another 12-week, open-label study also demonstrated improved adherence as well as reduced IOP and ocular surface disease upon switching to BAC-free travoprost. ${ }^{51}$

PF $\beta$-blockers are also available, such as PF timolol $0.1 \%$ gel, which is effective in decreasing IOP in treatment-naïve patients, and in controlling $\mathrm{IOP}^{52}$ as well as reducing ocular signs and symptoms in patients intolerant to their previous preserved medications; this gel has been shown to cause fewer changes to the ocular surface by in vivo confocal microscopy. ${ }^{53}$ It should not be forgotten, however, that $\beta$-blockers have a less favourable systemic side-effect profile in comparison with prostaglandin analogues, and $\beta$-blockers are frequently contra-indicated.

PF latanoprost (Monopost ${ }^{\oplus}$, Thea Pharmaceuticals, Staffordshire, UK) uses an innovative formulation solution known as Protriaxin ${ }^{\oplus}$ (Thea Pharmaceuticals). Protriaxin contains a solvent polymer with surfactant properties and two different types of polymer able to form a matricial network by cross-linking, in which the mesh contains the drug substance. The innovative formulation ensures excellent stability of the drug 
product, even at room temperature, and prevents adsorption of the drug substance onto the plastic material of the single-dose unit containers. The addition of a gelling inhibitor allows the product to have a low viscosity, making administration of the drops easier for patients. Monopost is also isotonic and has a neutral $\mathrm{pH}$, so that the product is less likely to sting in comparison with many other topical anti-glaucomatous therapies.

Direct comparative studies are limited; however, it has been shown in cultured human corneal epithelial cells, that PF tafluprost caused a significant reduction in cell viability compared with PF latanoprost, as well as greater in vivo irritation in rabbit eyes..$^{54}$ In contrast, whilst PF latanoprost is effective and seems to show fewer ocular side effects, there is some evidence to show potential superiority of other PF prostaglandins over latanoprost. For example, a multicentre trial showed superior efficacy for PF bimatoprost over PF latanoprost at lowering IOP at 6 months. ${ }^{55}$

The RELIEF study assessed the tolerability and efficacy following changing from BAC-preserved latanoprost to PF latanoprost in 140 patients with glaucoma or ocular hypertension. ${ }^{56}$ Mean IOP remained stable after 90 days at $15.9 \mathrm{mmHg}(\mathrm{p}<0.006)$. Moderate-to-severe conjunctival hyperaemia decreased from $56.8 \%$ to $1.6 \%$, and after 90 days there was a change in subjective assessment of tolerability from a mean score of 5.3 at day 0 to 1.9 at day $90(p<0.0001)$. Thus, patients had a similar IOP-lowering effect with the PF latanoprost, but with a better degree of tolerability.

A phase III multicentre trial across 87 centres comparing the efficacy of PF latanoprost (Monopost) versus preserved latanoprost (e.g., Xalatan ${ }^{\circledR}$, Pfizer, New York, NY, USA) in 404 patients, was conducted in 2013.57 Briefly, the cohort consisted of patients with POAG or ocular hypertension previously managed with BAC-preserved latanoprost who were randomised to therapy with either PF or preserved latanoprost for 3 months. There was a run-in phase with brinzolamide for 5 weeks followed by a washout phase of 5 days. Efficacy in reducing IOP was comparable between the two groups after 84 days $(-8.6 \pm 2.6 \mathrm{mmHg}$ $[-36 \%]$ for the PF version versus $-9.0 \pm 2.4 \mathrm{mmHg}[-38 \%]$ for the preserved version). Ocular adverse effects were reported in 18 (8.5\%) patients on the PF version versus 22 (11.6\%) patients on the preserved version. The most frequent ocular adverse event, drug intolerance, was reported in one $(0.5 \%)$ patient on the PF version versus four (2.1\%) patients on the preserved version. Moderate-to-severe conjunctival hyperaemia was less frequent with the PF version than with the preserved version at day 42 (20.2\% versus $30.6 \% ; p=0.003)$ and day 84 (21.4\% versus 29.1\%; $p=0.02$ ). The Global Symptoms Score, which includes pruritis, burning/stinging sensation, dry eye or foreign body sensation, was significantly lower with the PF version than the preserved version on day 42 ( 0.15 versus $0.41 ; p=0.001$ ) and day 84 ( 0.18 versus $0.46 ; p=0.001$ ). Overall, the results suggested a similar efficacy between the two products but a better tolerability with the PF version.

Since there is now a choice of three PF topical prostaglandin analogue anti-glaucomatous agents, it is important to know which of the products should be chosen as an ideal therapy for an individual patient. The drug of choice should, of course, be sufficiently efficacious and well-tolerated, inducing minimal, if any, adverse effects. In addition, the cost of the product prescribed should be taken into account to minimise any unnecessary healthcare expenditure (Table 1). It can be seen that PF latanoprost is the least expensive option out of the PF prostaglandin analogue agents. In a well-resourced health system, the single-use PF option may be the most cost-effective. In contrast, in developing countries, other agents (which contain preservatives) may
Table 1:

Drug tariff price of 30-pack size preservative-free agents

\begin{tabular}{|l|l|l|l|}
\hline Drug & Lumigan & Monopost & \\
& ( & Saflutan $^{\circledR}$ \\
\hline Active ingredient & Bimatoprost & Latanoprost & Tafluprost \\
\hline Concentration, $\mu / \mathrm{mL}$ & 300 & 50 & 15 \\
\hline Unit dose, $\mathrm{mL}$ & 0.4 & 0.2 & 0.3 \\
\hline Price, $\mathrm{f}$ & 13.75 & 8.49 & 12.20 \\
\hline
\end{tabular}

indeed be more affordable. Alone, yearly therapy with unoprostone is the least expensive prostaglandin analogue (approximately \$180) with bimatoprost, latanoprost and travoprost all having comparable prices (approximately $\$ 211-230$ ). ${ }^{58}$

Individual patients may respond to and/or tolerate one particular product better than another and this should be taken into account when managing a patient. PF latanoprost has equal efficacy to BAC-preserved latanoprost but with fewer side effects. ${ }^{59} \mathrm{~A}$ meta-analysis of 21 studies showed that PF latanoprost was statistically equally as effective as other prostaglandin analogues (including BAC-preserved latanoprost) at reducing IOP whilst showing statistically significant superiority over PF tafluprost. ${ }^{16}$ The risk of hyperaemia, however, was statistically significantly less with PF latanoprost compared with all other prostaglandin analogues, indicating that it might be the better option to offer patients. ${ }^{60}$

Another final issue to be taken into account is ease of administration of a drug. Many patients suffering from glaucoma are elderly with psychomotor difficulties; they may prefer a particular type of bottle format. Although it might be assumed that elderly patients would prefer larger multi-dose bottles, a study found that single-dose units were favoured over larger multi-dose bottles. ${ }^{61}$ Although most PF prostaglandin therapies are only available in single-dose units, PF bimatoprost is now available as either single-dose units (Lumigan) or a multi-dose bottle (Eyreida), meaning that patient choice is available.

\section{Conclusion}

Glaucoma will continue to cause significant global ocular morbidity and blindness in the foreseeable future. As such, it is of paramount importance that it is treated appropriately and effectively. Medically, this means using the best possible topical medication with the fewest side effects. The use of preservatives such as BAC may have potential (unproven) benefits, but for many patients can lead to the development of a symptomatic ocular surface disorder, intolerability and consequently, poor adherence. Furthermore, even sub-clinical changes to the conjunctiva may have adverse effects on post-operative wound healing, risking failure of any potential future glaucoma filtration surgery.

Prostaglandin antagonists remain the first-line anti-glaucoma drug for many practitioners, and their side effects cannot be overlooked especially until a more efficacious drug can be found. PF therapy thus presents a highly appealing choice. However, it should be noted that in financially-challenged health systems, such therapy may be uneconomical. Whilst there are several PF agents with few ocular side effects, we do believe a cost-effectiveness analysis is important in choosing the correct agent especially in countries with limited healthcare resources. We propose that clinicians should have a relatively low threshold in considering the use of PF monotherapeutic agents to minimise undesirable effects, improve adherence and still provide an efficacious treatment to control IOP in patients with glaucoma or significant ocular hypertension. $\square$ 
1. Quigley HA. Glaucoma. Lancet. 2011;377:1367-77.

2. Hodge WG, Lachaine J, Steffensen I, et al. The efficacy and harm of prostaglandin analogues for IOP reduction inglaucoma patients compared to dorzolamide and brimonidine: a systematic review. Br I Ophthalmol. 2008:92:7-12.

3. van der Valk R, Webers CA, Schouten JS, et al. Intraocular pressure-lowering effects of all commonly used glaucoma drugs: a meta-analysis of randomized clinical trials. Ophthalmology. 2005;112:1177-85.

4. Bean GW, Camras CB. Commercially available prostaglandin analogs for the reduction of intraocular pressure: similarities and differences. Surv Ophthalmol. 2008;53:69-84.

5. European Glaucoma Society Terminology and Guidelines for Glaucoma, 4th Edition - Chapter 3: Treatment principles and options. Supported by the EGS Foundation. Br J Ophthalmol. 2017; 101:130-95

6. Aguayo Bonniard A, Yeung JY, Chan CC, Birt CM. Ocular surface toxicity from glaucoma topical medications and associated preservatives such as benzalkonium chloride (BAK). Expert Opin Drug Metab Toxicol. 2016;12:1279-89.

7. Kaur IP, Lal S, Rana C, et al. Ocular preservatives: associated risks and newer options. Cutan Ocul Toxicol. 2009:28:93-103.

8. Baudouin C, Denoyer A, Desbenoit N, et al. In vitro and in vivo experimental studies on trabecular meshwork degeneration induced by benzalkonium chloride (an American Ophthalmological Society thesis). Trans Am Ophthalmol Soc. 2012;110:40-63.

9. Chen W, Zhang Z, Hu J, et al. Changes in rabbit corneal innervation induced by the topical application of benzalkonium chloride. Cornea. 2013;32:1599-606.

10. Li F, Huang W, Zhang X. Efficacy and safety of different regimens for primary open-angle glaucoma or ocular hypertension: a systematic review and network meta-analysis. Acta Ophthalmol. 2018:96:e277-84.

11. Li T, Lindsley $K$, Rouse B, et al. Comparative effectiveness of first-line medications for primary open-angle glaucoma: a systematic review and network meta-analysis. Ophthalmology. 2016:123:129-40.

12. Digiuni $M$, Fogagnolo $P$, Rossetti L. A review of the use of latanoprost for glaucoma since its launch. Expert Opin Pharmacother. 2012;13:723-45.

13. Parrish RK, Palmberg P, Sheu WP; XLT Study Group. A comparison of latanoprost, bimatoprost, and travoprost in patients with elevated intraocular pressure: a 12-week, randomized, masked-evaluator multicenter study. Am J Ophthalmol. 2003;135:688-703.

14. Susanna R Jr, Giampani J Jr, Borges AS, et al. A double-masked, randomized clinical trial comparing latanoprost with unoprostone in patients with open-angle glaucoma or ocular hypertension. Ophthalmology. 2001;108:259-63.

15. Takemoto $D$, Higashide $T$, Saito $Y$, et al. Intraocular pressure and visual field changes in normal-tension glaucoma patients treated using either unoprostone or latanoprost: a prospective comparative study. Clin Ophthalmol. a prospective con

16. Cucherat M, Stalmans I, Rouland JF. Relative efficacy and safety of preservative free latanoprost (T2345) for the treatment of open-angle glaucoma and ocular hypertension: an adjusted Indirect comparison meta-analysis of randomized clinical trials. J Glaucoma. 2014;23:69-75

17. Mishra D, Sinha BP, Kumar MS. Comparing the efficacy of latanoprost $(0.005 \%)$, bimatoprost $(0.03 \%)$, travoprost $(0.004 \%)$ and timolol $(0.5 \%)$ in the treatment of primary open angle glaucoma. Korean J Ophthalmol. 2014;28:399-407.

18. Pauly A, Brignole-Baudouin $F$, Guenoun JM, et al. Comparative study of topical anti-allergic eye drops on human conjunctiva-derived cells: responses to histamine and IFN gamma and toxicological profiles. Graefes Arch Clin Exp Ophthalmol. 2007:245:534-46.

19. Buron N, Micheau O, Cathelin S, et al. Differential mechanisms of conjunctival cell death induction by ultraviolet irradiation and benzalkonium chloride. Invest Ophthalmol Vis Sci. 2006;47:4221-30.

20. Broadway DC, Grierson I, O'Brien C, Hitchings RA. Adverse effects of topical antiglaucoma medication. II. The outcome of filtration surgery. Arch Ophthalmol. 1994;112:1446-54.

21. Schwab IR, Linberg JV, Gioia VM, et al. Foreshortening of the inferior conjunctival fornix associated with chronic glaucoma medications. Ophthalmology. 1992;99:197-202.

22. Noecker R. Effects of common ophthalmic preservatives on ocular health. Adv Ther. 2001;18:205-15.

23. Hamard P, Blondin C, Debbasch C, et al. In vitro effects of preserved and unpreserved antiglaucoma drugs on apoptotic marker expression by human trabecular cells. Graefes Arch Clin Exp Ophthalmol. 2003;241:1037-43.

24. Honrubia F, García-Sánchez J, Polo V, et al. Conjunctiva hyperaemia with the use of latanoprost versus other prostaglandin analogues in patients with ocular hypertension or glaucoma: a meta-analysis of randomised clinical trials. Br J Ophthalmol. 2009;93:316-21.

25. Kammer JA, Katzman B, Ackerman SL, Hollander DA. Efficacy and tolerability of bimatoprost versus travoprost in patients previously on latanoprost: a 3-month, randomised, masked-evaluator, multicentre study. Br J Ophthalmol 2010;94:74-9.

26. Efron N. Contact Lens Complications, Fourth Edition. Philadelphia, PA, USA: Elsevier, Ltd. 2019.

27. Shen W, Huang B, Yang J. Ocular surface changes in prostaglandin analogue-treated patients. J Ophthalmo. 2019;2019:9798272.

28. Jaenen N, Baudouin C, Pouliquen P, et al. Ocular symptoms and signs with preserved and preservative-free glaucoma medications. Eur J Ophthalmol. 2007;17:341-9.

29. Pisella PJ, Pouliquen P, Baudouin C. Prevalence of ocular symptoms and signs with preserved and preservative free glaucoma medication. Br J Ophthalmol. 2002;86:418-23.

30. Martone G, Frezzotti P, Tosi GM, et al. An in vivo confocal microscopy analysis of effects of topical antiglaucoma therapy with preservative on corneal innervation and morphology. Am J Ophthalmol. 2009;147:725-35.

31. Ichijima H, Petroll WM, Jester JV, Cavanagh HD. Confocal microscopic studies of living rabbit cornea treated with benzalkonium chloride. Cornea. 1992;11:221-5.

32. Yu FF, Liu X, Zhong YM, et al. Protection effect of sodium hyaluronate on ocular surface toxicity induced by benzalkonium chloride-preserved brimonidine in rabbits. Zhonghua Yan Ke Za Zhi. 2013;49:973-80.

33. Noecker RJ, Herrygers LA, Anwaruddin R. Corneal and conjunctival changes caused by commonly used glaucoma medications. Cornea. 2004:23:490-6.

34. Ha JY, Sung MS, Park SW. Effects of preservative on the meibomian gland in glaucoma patients treated with prostaglandin analogues. Chonnam Med J. 2019;55:156-62.

35. Chung SH, Lee SK, Cristol SM, et al. Impact of short-term exposure of commercial eyedrops preserved with benzalkonium chloride on precorneal mucin. Mol Vis. 2006:12:415-21.

36. Imayasu M, Moriyama T, Ohashi J, et al. A quantitative method for $\mathrm{LDH}, \mathrm{MDH}$ and albumin levels in tears with ocular surface toxicity scored by Draize criteria in rabbit eyes. CLAO $\mathrm{J}$. 1992:18:260-6.

37. Collin $\mathrm{HB}$, Grabsch BE. The effect of ophthalmic preservatives on the healing rate of the rabbit corneal epithelium after keratectomy. Am J Optom Physiol Opt. 1982;59:215-22.

38. Broadway DC, Chang LP. Trabeculectomy, risk factors for failure and the preoperative state of the conjunctiva. J Glaucoma. 2001;10:237-49

39. Reardon G, Kotak S, Schwartz GF. Objective assessment of compliance and persistence among patients treated for glaucoma and ocular hypertension: a systematic review. Patient Prefer Adherence. 2011;5:441-63.

40. Zimmerman TJ, Hahn SR, Gelb L, et al. The impact of ocular adverse effects in patients treated with topical prostaglandin analogs: changes in prescription patterns and patient persistence. J Ocul Pharmacol Ther. 2009;25:145-52.

41. Jaenen N, Baudouin C, Pouliquen P, et al. Ocular symptoms and signs with preserved and preservative-free glaucoma medications. Eur J Ophthalmol. 2007;17:341-9.

42. Hommer A, Mohammed Ramez O, Burchert M, Kimmich F. IOP-lowering efficacy and tolerability of preservative-free tafluprost $0.0015 \%$ among patients with ocular hypertension or glaucoma. Curr Med Res Opin. 2010;26:1905-13.

43. Lee W, Lee $\mathrm{S}$, Bae $\mathrm{H}$, et al. Efficacy and tolerability of preservative-free $0.0015 \%$ tafluprost in glaucoma patients: a prospective crossover study. BMC Ophthalmol. 2017;17:61.

44. Chabi A, Baranak C, Lupinacci R, Herring WJ. Preservative-free tafluprost in the treatment of open-angle glaucoma or ocular hypertension in India: a phase III clinical trial. Int I Clin Pract. 2016;70:577-86

45. Lanzl I, Hamacher T, Rosbach K, Ramez MO, et al. Preservative-free tafluprost in the treatment of naive patients with glaucoma and ocular hypertension. Clin Ophthalmol. 2013;7:901-10

46. Uusitalo H, Egorov E, Kaarniranta K, et al. Benefits of switching from latanoprost to preservative-free tafluprost eye drops: a meta-analysis of two phase IIIb clinical trials. Clin Ophthalmol. 2016;10:445-54

47. Karhanová M, Mlčák P, Fryšák Z, Marešová K. Efficacy and tolerability of preservative-free tafluprost $0.0015 \%$ in the treatment of glaucoma and ocular hypertension. Cesk Slov treatment of glaucoma and

48. Erb C, Lanzl I, Seidova SF, Kimmich F. Preservative-free tafluprost $0.0015 \%$ in the treatment of patients with glaucoma and ocular hypertension. Adv Ther. 2011;28:575-85

49. Pillunat LE, Eschstruth P, Häsemeyer S, Thelen U, et al. Preservative-free bimatoprost $0.03 \%$ in patients with primary open-angle glaucoma or ocular hypertension in clinical practice. Clin Ophthalmol. 2016;10:1759-65.

50. Henry JC, Peace JH, Stewart JA, Stewart WC. Efficacy, safety, and improved tolerability of travoprost BAK-free ophthalmic solution compared with prior prostaglandin therapy. Clin Ophthalmol. 2008:2:613-21.

51. García-Feiloo J, Muñoz-Negrete FJ, Hubatsch DA, Rossi GC Efficacy and tolerability of benzalkonium chloride-free travoprost in glaucoma patients switched from benzalkonium chloride-preserved latanoprost or bimatoprost. Clin Ophthalmo 2016;10:2085-91

52. Lazreg S, Merad Z, Nouri MT, et al. Efficacy and safety of preservative-free timolol $0.1 \%$ gel in open-angle glaucoma and ocular hypertension in treatment-naiive patients and patients intolerant to other hypotensive medications. J Fr Ophtalmol. 2018;41:945-54

53. Frezzotti P, Fogagnolo P, Haka G, et al. In vivo confocal microscopy of conjunctiva in preservative-free timolol $0.1 \%$ gel formulation therapy for glaucoma. Acta Ophthalmol. 2014;92:133-40.

54. Esaki Y, Shimazaki A, Pellinen P. Ocular tolerability of preservative-free tafluprost and latanoprost: in vitro and in vivo comparative study. Open Ophthalmol J. 2016;10:146-53.

55. Stalmans I Oddone F Cordeiro MF et al. Comparison of preservative-free latanoprost and preservative-free bimatoprost in a multicenter, randomized, investigator-masked cross-over clinical trial, the SPORT trial. Graefes Arch Clin Exp ophthalmol. 2016:254:1151-8.

56. Misiuk-Hojlo M, Pomorska M, Mulak M, et al. The RELIEF study: Tolerability and efficacy of preservative-free latanoprost in the treatment of glaucoma or ocular hypertension. Eur J Ophthalmol. 2018;29:210-5.

57. Rouland JF, Traverso CE, Stalmans I, Fekih LE, et al. T2345 Study Group. Efficacy and safety of preservative-free latanoprost eyedrops, compared with BAK-preserved latanoprost in patients with ocular hypertension or glaucoma. Br J Ophthalmol. 2013;97:196-200.

58. Mick AB, Gonzalez S, Dunbar MT, McSoley JJ. A cost analysis of the prostaglandin analogs. Optometry. 2002;73:614-9.

59. Sanford M. Preservative-free latanoprost eye drops in patients with primary open-angle glaucoma/ocular hypertension. Clin Drug Investig. 2014:34:521-8.

60. Feldman RM. Conjunctival hyperemia and the use of topical prostaglandins in glaucoma and ocular hypertension. J Ocul Pharmacol Ther. 2003;19:23-35.

61. Parkkari M, Latvala T, Ropo A. Handling test of eye drop dispenser-comparison of unit-dose pipettes with conventional eye drop bottles. J Ocul Pharmacol Ther. 2010;26:273-6. 